\title{
UNIQUENESS OF APERIODIC KNEADING SEQUENCES
}

\author{
KAREN M. BRUCKS
}

(Communicated by R. Daniel Mauldin)

\begin{abstract}
The trapezoidal function $f_{e}(x)$ is defined for fixed $e \in(0,1 / 2)$ by $f_{e}(x)=(1 / e) x$ for $x \in[0, e], f_{e}(x)=1$ for $x \in(e, 1-e)$, and $f_{e}(x)=$ $(1 / e)(1-x)$ for $x \in[1-e, 1]$. For a given $e$ and the associated one-parameter family of maps $\left\{\lambda f_{e}(x) \mid \lambda \in[0,1]\right\}$, we show that if $A$ is an aperiodic kneading sequence, then there is a unique $\lambda \in[0,1]$ so that the itinerary of $\lambda$ under the map $\lambda f_{e}$ is $A$. From this, we conclude that the "stable windows" are dense in $[0,1]$ for the one-parameter family $\lambda f_{e}$.
\end{abstract}

This note is mainly concerned with those maps which are trapezoidal. The trapezoidal function, $f_{e}$, is defined for $e \in(0,1 / 2)$ by $f_{e}(x)=x / e$ for $x \in$ $[0, e], f_{e}(x)=1$ for $x \in(e, 1-e)$, and $f_{e}(x)=(1-x) / e$ for $x \in[1-e, 1]$. For a fixed $e$, one can form a one parameter family of maps by considering the set $\left\{\lambda f_{e} \mid \lambda \in[0,1]\right\}$.

Throughout this note, the notation and terminology of Beyer, Mauldin, and Stein (BMS) [2] is used. Thus, if $g$ maps $[0,1]$ into itself and $x \in[0,1]$, then the itinerary of $x$ under $g$ is given by $I^{g}(x)=\left\{b_{i}\right\}_{i \geq 0}$, where $b_{i}=R$ if $g^{i}(x)>1 / 2, b_{i}=L$ if $g^{i}(x)<1 / 2$, and $b_{i}=C$ if $g^{i}(x)=1 / 2$ (if $b_{i}=C$ for some $i$, then the itinerary stops). We note that $I^{g}(x)$ is either an infinite sequence of $R$ 's and $L$ 's or is a finite sequence of $R$ 's and $L$ 's followed by a $C$. If $g$ is unimodal and $\lambda \in[0,1]$ is such that the orbit of $\lambda$ under the scaled map $\lambda g$ contains $1 / 2$, then the finite sequence $I^{\lambda g}(\lambda)$ is referred to as an MSS sequence [2, 9].

For $g$ unimodal and $\lambda \in[0,1]$, the intinerary of $\lambda$ under the map $\lambda g$, $I^{\lambda g}(\lambda)$, is referred to as the kneading sequence of $\lambda g[6]$. BMS show that $I^{\lambda g}(\lambda)$ is shift maximal in the parity-lexicographical order when $g$ is unimodal and $\lambda \in[0,1]$ (throughout this note, when comparing kneading sequences the paritylexicographical order is used). Furthermore, if $B$ is a finite or infinite shift maximal sequence, then there is some unimodal map $g$ and some $\lambda \in[0,1]$ so that $I^{\lambda g}(\lambda)=B$. Thus any kneading sequence is shift maximal, and any shift maximal sequence is a kneading sequence. We note that if $g$ is unimodal and

Received by the editors December 1, 1988 and, in revised form, January 25, 1989.

1980 Mathematics Subject Classification (1985 Revision). Primary 26A18; Secondary 39B10. 
$\lambda \in[0,1]$, then $I^{\lambda g}(\lambda)$ is either an MSS sequence, or is infinite and periodic, or is infinite and aperiodic. In this note we prove the following theorem.

Theorem A. Fix $e \in(0,1 / 2)$. Let $A$ be aperiodic shift maximal sequence. Then there exists a unique $\lambda \in[0,1]$ such that $I^{\lambda f_{e}}(\lambda)=A$.

Let us comment on our interest in Theorem A in general terms. For a unimodal map $g$ let

$$
\mathscr{P}_{g}=\left\{\lambda \in[0,1] \mid I^{\lambda g}(\lambda) \text { is an MSS sequence or is infinite and periodic }\right\} .
$$

It is widely believed $\left[7 ; 4\right.$, pp. 31,69] that $\mathscr{P}_{4 x(1-x)}$ is dense in $[0,1]$. We note that $\mathscr{P}_{4 x(1-x)}$ consists of precisely those $\lambda$ such that $\lambda 4 x(1-x)$ has a stable orbit [4, p. 69]. Similarly, $\mathscr{P}_{f_{e}}$ consists of those values of $\lambda$ such that $\lambda f_{e}$ has a stable orbit or a finite orbit containing either $e$ or $1-e$. Theorem $\mathrm{A}$ and Lemma 1.1 (of Appendix A) imply that $\mathscr{P}_{f_{e}}$ is dense in $[0,1]$, i.e., the "stable windows" are dense in $[0,1]$. If one could prove Theorem $A$ for the family $\lambda 4 x(1-x)$, then again the "stable windows" would be dense in $[0,1]$. Note that there are uncountably many aperiodic shift maximal sequences.

We say that the one-parameter family $\{\lambda g \mid \lambda \in[0,1]\}$, where $g$ is unimodal, exhibits uniqueness provided that for each MSS sequence $P$ there exists exactly one $\lambda$ such that $I^{\lambda g}(\lambda)=P$. Moreover, the family $\{\lambda g \mid \lambda \in[0,1]\}$ is said to be fully unique if it exhibits uniqueness and if for each aperiodic kneading sequence $A$ there is exactly one $\lambda$ such that $I^{\lambda g}(\lambda)=A$. Fix $e \in(0,1 / 2)$. It is known that $\left\{\lambda f_{e} \mid \lambda \in[0,1]\right\}$ and $\{\lambda 4 x(1-x) \mid \lambda \in[0,1]\}$ exhibit uniqueness $[2,8,10]$. In this note, we establish that the family $\left\{\lambda f_{e}\right\}$ is fully unique. We remark that this is the only one-parameter family shown to be fully unique. If we take $g(x)$ to be $4 x(1-x)$, then for certain but not all aperiodic kneading sequences Dennis Sullivan has shown that there is a unique $\lambda$ so that $I^{\lambda g}(\lambda)$ is the given kneading sequence [11]. Moreover he has shown that if there exists an analytic family that is fully unique, then the family $\lambda 4 x(1-x)$ is fully unique.[11].

This paper is broken into two sections. Section one consists of general observations, and preliminary comments. Section two contains the proof of Theorem A.

BMS [2] show that the one-parameter family $\lambda f_{e}$ exhibits uniqueness for $0<$ $e<(3 \operatorname{sqrt}(17)-11) / 4$ (we note that $(3 \operatorname{sqrt}(17)-11) / 4 \cong 0.3423)$. Metropolis and Louck [8] show uniqueness for any $e \in(0,1 / 2)$. Our proof of Theorem A uses uniqueness for a given $e$. If $e \in(0,1 / 2)$ and $B$ is some periodic shift maximal sequence, it is known that there is more than one $\lambda$ with $I^{\lambda f_{e}}(\lambda)=B$. This will be discussed presently. Throughout the rest of this note assume that $e \in(0,1 / 2)$ is fixed. We state the following fact as a theorem and outline its proof in Appendix $A$. 
Theorem B. Let $g$ be a unimodal Lipschitz continuous concave function that has a continuous derivative in a neighborhood of $x=1 / 2$. Furthermore assume that $g$ exhibits uniqueness. Then the following hold.

(i) If $B$ is an aperiodic shift maximal sequence, then $\left\{\lambda \in[0,1] \mid I^{\lambda g}(\lambda)=\right.$ $B\}$ is a closed interval or consists of a single point.

(ii) If $B=\left\{b_{i}\right\}_{i \geq 1}$ is a periodic shift maximal sequence of period $k$, then exactly one of the following hold.

(a) The sequence $b_{1} \cdots b_{k-1} C$ is the harmonic of some MSS sequence $P$, and the set of $\lambda$ for which $I^{\lambda g}(\lambda)=B$ is an open interval.

(b) The sequence $b_{1} \cdots b_{k-1} C$ is not the harmonic of some MSS sequence, and the set of $\lambda$ for which $I^{\lambda g}(\lambda)=B$ is either an open or half open interval. More precisely, if $b_{1} \cdots b_{k-1}$ is odd (even), then $\left(b_{1} \cdots b_{k-1} R\right)^{\infty}$ $\left(\left(b_{1} \cdots b_{k-1} L\right)^{\infty}\right)$ is a left closed right open interval, and $\left(b_{1} \cdots b_{k-1} L\right)^{\infty}$ $\cdot\left(\left(b_{1} \cdots b_{k-1} R\right)^{\infty}\right)$ is an open interval.

Recall that $e \in(0,1 / 2)$ is fixed. Set $f=f_{e}$. For $x$ in $\mathbf{R}$ and $\lambda \in(0,1]$ set

$$
f_{\lambda, R}^{-1}(x)=1-(e / \lambda) x
$$

and

$$
f_{\lambda, L}^{-1}(x)=(e / \lambda) x
$$

For $P=P_{1} \cdots P_{n} \in\{R, L\}^{n}$ set

(i) $\rho(P)=\left\{j \mid P_{j}=R\right\}$, and

(ii) $G_{\lambda}(P, y)=f_{\lambda, P_{1}}^{-1}\left(f_{\lambda, P_{2}}^{-1}\left(\cdots\left(f_{\lambda, P_{n}}^{-1}(y)\right) \cdots\right)\right)$.

Beyer and Ebanks [1] have the following theorem, which can be proved by induction on $n$.

Theorem 2.1. Let $P=P_{1} \cdots P_{n}$ be a finite sequence of $R$ 's and $L$ 's. Then,

$$
\begin{aligned}
G_{\lambda}(P, y)= & \sum_{j \in \rho(P)}(-1)^{\left|\rho\left(P_{1} \cdots P_{j}\right)\right|-1}(e / \lambda)^{j-1} \\
& +(-1)^{|\rho(P)|}(e / \lambda)^{|P|} y .
\end{aligned}
$$

Remark 2.2. Let $A=\left\{a_{i}\right\}_{i \geq 1}$ be an aperiodic shift maximal sequence, and for each $n \in \mathbf{N}$ let $\left.A\right|_{n}=\left\langle a_{1}, \ldots, a_{n}\right\rangle$. Suppose that $I^{\lambda f}(\lambda)=A$. Observe the following two facts.

(i) For each $n \in \mathbf{N},(\lambda f)^{n}(\lambda) \notin[e, 1-e]$, for otherwise $A$ would be periodic.

(ii) Fix $n \in \mathbf{N}$. Then $G_{\lambda}\left(\left.A\right|_{n},(\lambda f)^{n}(\lambda)\right)=\lambda$. To see this note that $\lambda \rightarrow(\lambda f)(\lambda)=\lambda f_{a_{1}}(\lambda) \rightarrow(\lambda f)^{2}(\lambda)=\lambda f_{a_{2}}\left(\lambda f_{a_{1}}(\lambda)\right) \rightarrow \cdots \rightarrow(\lambda f)^{n}(\lambda)=$ $\left(\lambda f_{a_{n}}\left(\cdots\left(\lambda f_{a_{1}}(\lambda)\right) \cdots\right)\right)$, where $f_{L}(x)=(1 / e) x$ and $f_{R}(x)=(1 / e)(1-$ $x)$, with $(\lambda f)^{j}(\lambda) \notin[e, 1-e]$ for $1 \leq j \leq n$. 
Remark 2.3. For $P=R L^{n}$, let $\lambda_{n}$ be the unique scalar such that $I^{\lambda_{n} f}\left(\lambda_{n}\right)=P$. BMS show that $\lambda_{n} \rightarrow 1$ as $n \rightarrow \infty$. Thus Theorem $A$ holds if $A$ is $R L^{\infty}$.

Proof of Theorem A. Recall that $A$ is an aperiodic shift maximal sequence. We assume, using Remark 2.3, that $A \neq R L^{\infty}$. Suppose there is more than one $\lambda$ such that $I^{\lambda f}(\lambda)=A$. Then Theorem B implies that there exists a closed interval $[\alpha, \beta] \subset[0,1]$ such that for every $\lambda \in[\alpha, \beta]$ we have $I^{\lambda f}(\lambda)=A$. Note that $\alpha \geq(1-e)$. Again, say $A=\left\{a_{i}\right\}_{i \geq 1}$ and for each $n \in \mathbf{N}$ let $\left.A\right|_{n}$ denote the finite sequence $\left\langle a_{1}, \ldots, a_{n}\right\rangle$.

Let $\lambda \in[\alpha, \beta]$ and $n \in \mathbf{N}$. Then, as in Remark 2.2,

$$
G_{\lambda}\left(\left.A\right|_{n},(\lambda f)^{n}(\lambda)\right)=\lambda \text {. }
$$

Thus for every $\lambda \in[\alpha, \beta]$ and $n \in \mathbf{N}$ we have that (1) holds.

For $\lambda \in(e, 1]$ and $n \in \mathbf{N}$ set

$$
g_{\lambda, n}(x)=G_{\lambda}\left(\left.A\right|_{n},(\lambda f)^{n}(x)\right), \quad x \in[0,1] .
$$

Then,

$$
g_{\lambda, n}(\lambda)=\lambda \quad \text { for all } \lambda \in[\alpha, \beta] \text { and } n \in \mathbf{N} .
$$

Also, for each $\lambda \in(e, 1]$ set

$$
g(\lambda)=\sum_{j \in \rho(A)}(-1)^{\left|\rho\left(a_{1} \cdots a_{j}\right)\right|-1}(e / \lambda)^{j-1} .
$$

Notice that $g(\lambda)$ is the limit of the $g_{\lambda, n}(\lambda)$ as $n \rightarrow \infty$. We have $g(\lambda)$ and $g_{\lambda, n}(x)$ defined for $\lambda \in(e, 1], n \in \mathbf{N}$, and $x \in[0,1]$.

Next, fix $\lambda \in(e, 1], n \in \mathbf{N}$, and $x \in[0,1]$. Then,

$$
\begin{aligned}
g(\lambda)-g_{\lambda, n}(x)= & \sum_{\substack{j \in \rho(A) \\
j>n}}(-1)^{\left|\rho\left(a_{1} \cdots a_{j}\right)\right|^{-1}}(e / \lambda)^{j-1} \\
& -(-1)^{\left|\rho\left(\left.A\right|_{n}\right)\right|}(e / \lambda)^{n}(\lambda f)^{n}(x) .
\end{aligned}
$$

Thus,

$$
\left|g(\lambda)-g_{\lambda, n}(x)\right| \leq \sum_{\substack{j \in \rho(A) \\ j>n}}\left((e / \lambda)^{j-1}\right)+(e / \lambda)^{n} \lambda,
$$

for $\lambda \in(e, 1], n \in \mathbf{N}$, and $x \in[0,1]$.

Choose $\gamma<1$ so that $0<(e / \lambda)<\gamma$ for $\lambda \in[\alpha, \beta]$. Then for $\lambda \in[\alpha, \beta]$, $n \in \mathbf{N}$, and $x \in[0,1]$ we have

$$
\left|g(\lambda)-g_{\lambda, n}(x)\right| \leq \sum_{\substack{j \in \rho(A) \\ j>n}}\left(\gamma^{j-1}\right)+\gamma^{n} \beta .
$$

Hence, for every $\varepsilon>0$ there exists $m \in \mathbf{N}$ such that

$$
\left|g(\lambda)-g_{\lambda, m}(x)\right|<\varepsilon
$$

for all $\lambda \in[\alpha, \beta]$ and $x \in[0,1]$. 
In particular, for every $\varepsilon>0$ there exists $m \in \mathbf{N}$ so that

$$
|g(\lambda)-\lambda|=\left|g(\lambda)-g_{\lambda, m}(\lambda)\right|<\varepsilon
$$

for every $\lambda \in[\alpha, \beta]$. Thus,

$$
g(\lambda)=\lambda
$$

on $[\alpha, \beta]$.

However recall that

$$
g(\lambda)=\sum_{j \in \rho(A)}(-1)^{\left|\rho\left(a_{1} \cdots a_{j}\right)\right|-1}(e / \lambda)^{j-1},
$$

with $\rho(A)$ infinite. Setting $t=(e / \lambda)$ we find that

$$
\left(\sum_{j \in \rho(A)}(-1)^{\left|\rho\left(a_{1} \cdots a_{j}\right)\right|-1} t^{j}\right)-e=0
$$

on $[e / \beta, e / \alpha]$. This is a contradiction. Thus we have proven Theorem A.

\section{APPENDIX A}

The following lemmas and theorems are used to prove Theorem B.

Lemma 1.1. Let $f$ be unimodal, $0 \leq \lambda_{1}<\lambda_{2} \leq 1$, and $I^{\lambda_{1} f}\left(\lambda_{1}\right), I^{\lambda_{2} f}\left(\lambda_{2}\right)$ be distinct elements of $\{R, L\}^{\mathbf{N}}$. Then there is some $\lambda_{0} \in\left(\lambda_{1}, \lambda_{2}\right)$ such that $I^{\lambda_{0} f}\left(\lambda_{0}\right)$ is finite.

Proof. Let $k$ be the first index where $I^{\lambda_{1} f}\left(\lambda_{1}\right)$ and $I^{\lambda_{2} f}\left(\lambda_{2}\right)$ differ. Set

$\gamma=\sup \left\{\lambda \in\left(\lambda_{1}, \lambda_{2}\right) \mid I^{\lambda f}(\lambda)\right.$ agrees with $I^{\lambda_{1} f}\left(\lambda_{1}\right)$ in the first $k$ positions $\}$.

The $\lambda_{1}<\gamma<\lambda_{2}$, and $I^{\gamma f}(\gamma)$ is finite, since otherwise the definition of $\gamma$ is contradicted.

The following two theorems are taken from BMS.

Theorem 1.2. Let $f$ be a unimodal Lipschitz continuous function that has a continuous derivative in a neighborhood of $x=1 / 2$. Suppose $0 \leq \lambda_{1}<\lambda_{2} \leq 1$ and $A$ is a shift maximal sequence other than $L^{\infty}, C, R^{\infty}$, or $R L^{\infty}$. Suppose further that

$$
I^{\lambda_{1} f}\left(\lambda_{1}\right)<A<I^{\lambda_{2} f}\left(\lambda_{2}\right) .
$$

Then there exists some $\lambda \in\left(\lambda_{1}, \lambda_{2}\right)$ so that $I^{\lambda f}(\lambda)=A$. (The theorem also holds if $I^{\lambda_{1} f}\left(\lambda_{1}\right)>A>I^{\lambda_{2} f}\left(\lambda_{2}\right)$.)

Theorem 1.3. Let $f$ be a unimodal Lipschitz continuous concave function with a continuous derivative in a neighborhood of $1 / 2$. Then for each shift maximal sequence $P$ there is a value of $\lambda$ so that $I^{\lambda f}(\lambda)=P$.

In the proof of Theorem 1.2 BMS prove the following lemma. 
Lemma 1.4. Let $f$ be a unimodal Lipschitz continuous function that has a continuous derivative in a neighborhood of $x=1 / 2$. Suppose $\lambda_{0} \in[0,1]$ is such that $I^{\lambda_{0} f}\left(\lambda_{0}\right)$ is finite. Say, $I^{\lambda_{0} f}\left(\lambda_{0}\right)=P C$, where $P \in\{R, L\}^{n}$ for some $n$. Then there exists an open interval $U \subset[0,1]$ containing $\lambda_{0}$ so that if $\lambda$ is in $U$, then

$$
I^{\lambda f}(\lambda) \in\left\{P C,(P R)^{\infty},(P L)^{\infty}\right\} .
$$

Proof of Theorem B (i). We note that, by Theorem 1.3, there is some $\lambda$ with $I^{\lambda g}(\lambda)=B$. Let

$$
\beta=\sup \left\{\lambda \in[0,1] \mid I^{\lambda g}(\lambda)=B\right\},
$$

and

$$
\alpha=\inf \left\{\lambda \in[0,1] \mid I^{\lambda g}(\lambda)=B\right\} .
$$

Suppose that $\alpha<\beta$. Then Lemma 1.4 implies that both $I^{\alpha g}(\alpha)$ and $I^{\beta g}(\beta)$ are not finite and therefore must both be equal to $B$. If $I^{\lambda g}(\lambda)=B$ for all $\lambda \in[\alpha, \beta]$, then we are done. Suppose there is some $\lambda \in[\alpha, \beta]$ such that $I^{\lambda g}(\lambda) \neq B$. Then, by Lemma 1.1 , there exists some $\lambda_{0} \in[\alpha, \beta]$ such that $I^{\lambda_{0} g}\left(\lambda_{0}\right)=Q$ is finite.

Case 1. Suppose $Q>B$. Then, using Theorem 1.2, there is some $\lambda>\beta$ so that $Q=I^{\lambda g}(\lambda)$. This contradicts uniqueness.

Case 2. Suppose $Q<B$. The argument is similar to case one.

Remark 1.5. We briefly recall what the harmonics of an MSS sequence are. Let $P \in\{R, L\}^{k}$ be an MSS sequence; we have temporarily dropped the $C$. For $n \in \mathbf{N}$, set

$$
H_{n}(P)= \begin{cases}H_{n-1}(P) L H_{n-1}(P), & \text { if } H_{n-1}(P) \text { is odd, } \\ H_{n-1}(P) R H_{n-1}(P), & \text { if } H_{n-1}(P) \text { is even, }\end{cases}
$$

where $H_{0}(P)=P$.

Then $H_{n}(P)$ is called the $n$th harmonic of $P$. We let $H_{\infty}(P)$ be the unique element in $\{R, L\}^{\mathbf{N}}$ that is the common extension of the harmonics of $P$ and note that $H_{\infty}(P)$ is an aperiodic kneading sequence. Of course, $H_{m}(P)$ is odd (even) if there is an odd (even) number of $R$ 's in $H_{m}(P)$. The following facts are known $[5,9,3]$ (for (ii) see [3, Theorem 3.2, p. 436]).

(i) If $P$ is an MSS sequence and $Q$ is some shift maximal sequence so that $P<Q<H_{1}(P)$, then $Q=(P R)^{\infty}$ if $H_{1}(P)=P R P$ or $Q=(P L)^{\infty}$ if $H_{1}(P)=P L P$.

(ii) If $B=\left\{b_{i}\right\}_{i \geq 1}$ is a periodic shift maximal sequence of period $k$, then $b_{1} \cdots b_{k-1} C$ is an MSS sequence. Moreover, if $Q \neq b_{1} \cdots b_{k-1}$ is some MSS sequence with $a \in\{R, L\}$ so that $(Q a)^{\infty}=B$, then $Q$ is the first harmonic of $b_{1} \cdots b_{k-1} C$.

Proof of Theorem B (ii). Theorem B (ii) now follows from Remark 1.5, Lemma 1.4, and Theorem 1.2. 


\section{REFERENCES}

1. W. A. Beyer and B. R. Ebanks, Quadratic convergence of projections in period doubling for trapezoidal maps, preprint.

2. W. A. Beyer, R. D. Mauldin, and P. R. Stein, Shift-maximal sequences in function iteration: existence, uniqueness, and multiplicity, J. Math. Anal. Appl 115(1986), 305-362.

3. K. M. Brucks, MSS sequences, colorings of necklaces, and periodic points of $f(z)=z^{2}-2$, Adv. in App. Math. 8(1987), 434-445.

4. P. Collet and J.-P. Eckmann, Iterated maps on the interval as dynamical systems, Birkhauser, Basel, 1980.

5. B. Derrida, A. Gervois, and Y. Pomeau, Iteration of endomorphisms on the real axis and representations of numbers, Ann. Inst. Henri Poincare 29(1978), 305-356.

6. R. L. Devaney, An introduction to chaotic dynamical systems, Benjamin/Cummings Publishing Co., 1986.

7. C. Grebogi, E. Ott, and J. A. Yorke, Chaos, strange attractors, and fractal basin boundaries in nonlinear dynamics, Science 238(1987), 632-637.

8. J. D. Louck and N. Metropolis, Symbolic dynamics of trapezoidal maps, D. Reidel Publishing Company, 1986.

9. N. Metroplois, M. L. Stein, and P. R. Stein, On finite limit sets for transformations on the unit interval, J. Combin. Theory 15(1973), 25-44.

10. J. Milnor and W. Thurston, On iterated maps of the interval, Lect. Notes Math. 1342, Springer, 1988.

11. D. P. Sullivan, personal communications. 48824

Department of Mathematics, Michigan State University, East lansing, Michigan

Current address: Institute for Mathematical Sciences, State University of New York-Stony Brook, Stony Brook, New York, 11794-3660. 